\title{
Erratum: non-perturbative effects and Yukawa hierarchies in F-theory $\mathrm{SU}(5)$ unification
}

A. Font, ${ }^{a}$ L.E. Ibáñez, ${ }^{b, c}$ F. Marchesano ${ }^{b}$ and D. Regalado ${ }^{b, c}$

${ }^{a}$ Departamento de Física, Centro de Física Teórica y Computacional, Facultad de Ciencias, Universidad Central de Venezuela, A.P. 20513, Caracas 1020-A, Venezuela

${ }^{b}$ Instituto de Física Teórica UAM-CSIC,

C/ Nicolás Cabrera 13-15, Cantoblanco, 28049 Madrid, Spain

${ }^{c}$ Departamento de Física Teórica, Universidad Autónoma de Madrid, Campus de Cantoblanco, 28049 Madrid, Spain

E-mail: afont@fisica.ciens.ucv.ve, luis.ibannez@uam.es, fernando.marchesano@csic.es, diego.regalado@csic.es

ERRATUM TO: JHEP03(2013)140

KEYwords: F-Theory, Quark Masses and SM Parameters, Nonperturbative Effects, GUT

In sections 3 (except in subsection 3.3), 4-6 and appendices A-D, whenever the symbol $\mathcal{N}=$ appears in the text it should be replaced by the symbol $\neq$. 